\title{
Erratum: Constraints on the path-length dependence of jet quenching in nuclear collisions at RHIC and LHC
}

\author{
Barbara Betz $^{a}$ and Miklos Gyulassy ${ }^{b, c, d}$ \\ ${ }^{a}$ Institute for Theoretical Physics, Johann Wolfgang Goethe-University, \\ 60438 Frankfurt am Main, Germany \\ ${ }^{b}$ Department of Physics, Columbia University, \\ New York, 10027, U.S.A. \\ ${ }^{c}$ Nuclear Science Division, Lawrence Berkeley National Laboratory, \\ Berkeley, CA, U.S.A. \\ ${ }^{d}$ Institute for Particle and Nuclear Physics, Wigner RCP, \\ HAS, 1121 Budapest, Hungary \\ E-mail: betz@th.physik.uni-frankfurt.de, gyulassy@phys.columbia.edu
}

ERRATUM TO: JHEP08(2014)090

ARXIV EPRINT: 1404.6378

The figures 4 (b1) and (b2) and figures 9 (b1) and (b2) were calculated incorrectly. See replacement figures 1 and 2 below. The corrected figures imply that:

(a) The nuclear modification factor $R_{A A}$ at LHC energies for pure elastic energy loss [with $(a, b, c, q)=(0,0,2,-1)]$ in the new figure 1 is now found to be compatible with both RHIC and LHC energies for $\kappa_{\mathrm{RHIC}}=\kappa_{\mathrm{LHC}}$. The jet $v_{2}$-asymmetry is, however, still a factor of $\sim 2$ too low.

(b) The SLTc scenario assuming a radiative jet-energy loss coupling $\kappa(T)$ that is enhanced by a factor of three in the transition range of $113<T<173 \mathrm{MeV}$ [23-25] does in fact describe the LHC $R_{A A}$-data but is sensitive to the bulk hydrodynamic background temperature field. For this $\kappa(T)$ model the RL viscous hydro field [12, 13] is prefered by both $R_{A A}$ and $v_{2}$.

Open Access. This article is distributed under the terms of the Creative Commons Attribution License (CC-BY 4.0), which permits any use, distribution and reproduction in any medium, provided the original author(s) and source are credited. 

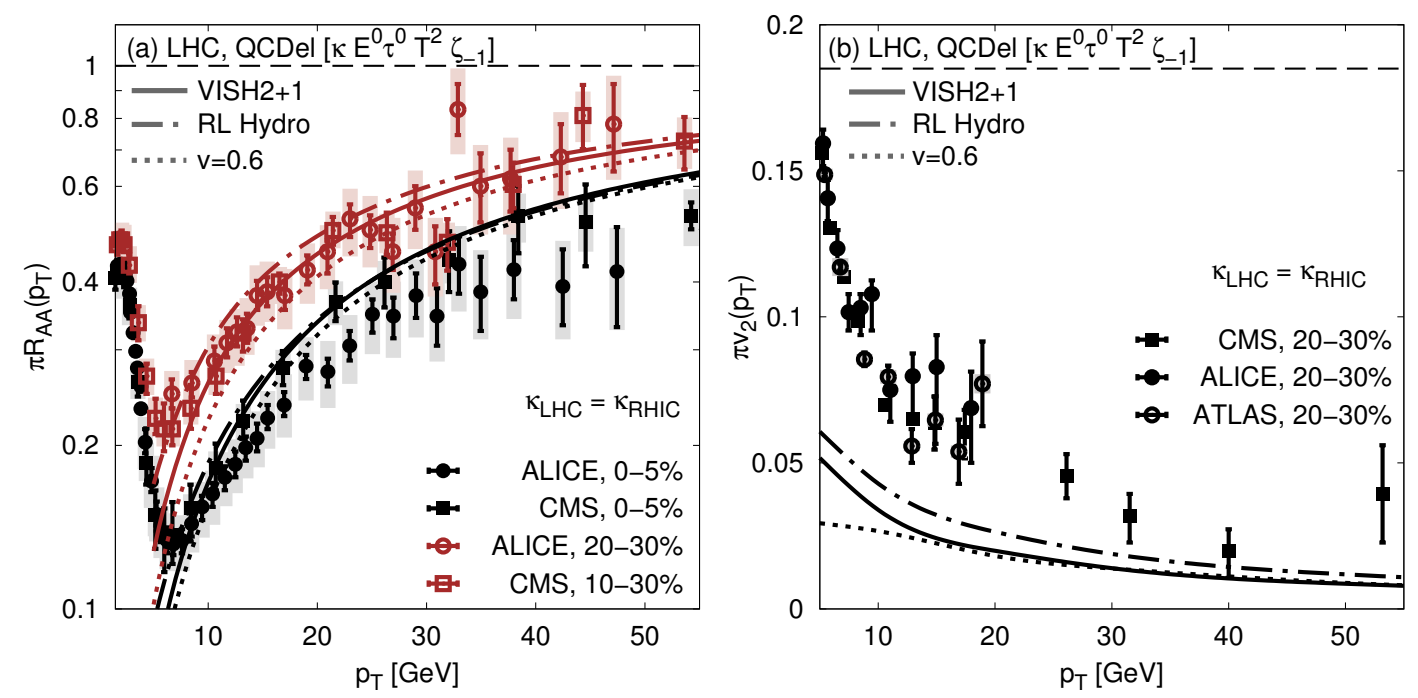

Figure 1. Corrected figure 4 (b1) and (b2) of the original publication. Panel (a) shows data for the pion nuclear modification factor $R_{A A}$ from ALICE [17] and CMS [19], while panel (b) depicts the high- $p_{T}$ elliptic flow as extracted from ALICE [18], CMS [20], and ATLAS [21, 22]. The model calculations assume elastic energy loss, $d E / d x=\kappa T^{2}$, with no energy-loss fluctuations using different bulk hydro temperature flow fields at LHC energies: viscous $\eta / s=0.08$ VISH2 $+1[9,10]$ (solid), viscous $\eta / s=0.08 \mathrm{RL}$ Hydro $[12,13]$ (dashed-dotted), and the $v_{\perp}=0.6$ blast wave model $[14,15]$ (dotted).
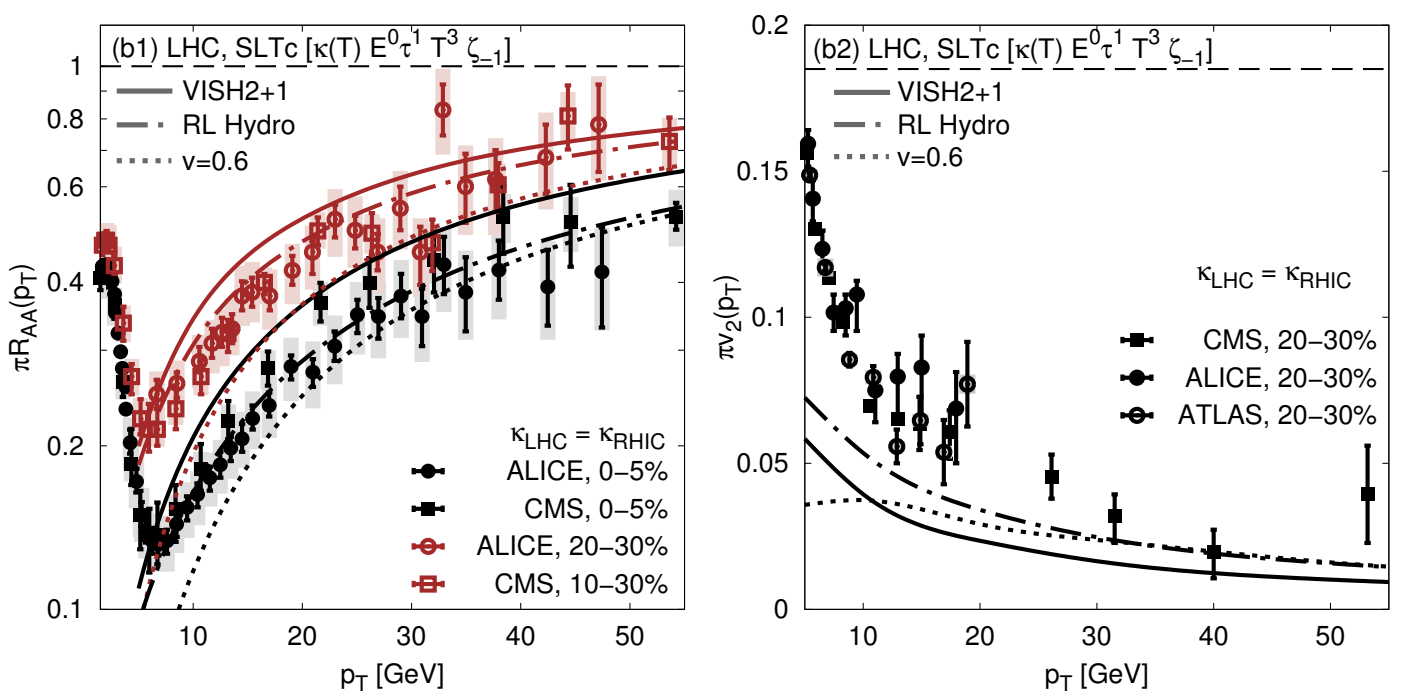

Figure 2. Corrected figure 9 (b1) and (b2) of the original publication. The pion nuclear modification factor $[17,19]$ and the high- $p_{T}$ elliptic flow $[18,20-22]$ are compared to the SLTc energy loss model, $d E / d x=\kappa(T) x T^{3}$, with enhanced coupling near $T_{c}$ [23-25], no energy-loss fluctuations, and different bulk QGP flow fields at LHC energies [9, 10, 12-15]. 


\section{References}

[9] C. Shen, U. Heinz, P. Huovinen and H. Song, Radial and elliptic flow in Pb+Pb collisions at the Large Hadron Collider from viscous hydrodynamic, Phys. Rev. C 84 (2011) 044903 [arXiv: 1105.3226] [INSPIRE].

[10] Z. Qiu, C. Shen and U. Heinz, Hydrodynamic elliptic and triangular flow in Pb-Pb collisions at $\sqrt{s}=2.76 \mathrm{TeV}$, Phys. Lett. B 707 (2012) 151 [arXiv:1110.3033] [INSPIRE].

[12] M. Luzum and P. Romatschke, Conformal relativistic viscous hydrodynamics: applications to RHIC results at $\sqrt{s_{\mathrm{NN}}}=200 \mathrm{GeV}$, Phys. Rev. C 78 (2008) 034915 [Erratum ibid. C 79 (2009) 039903] [arXiv:0804.4015] [INSPIRE].

[13] M. Luzum and P. Romatschke, Viscous hydrodynamic predictions for nuclear collisions at the LHC, Phys. Rev. Lett. 103 (2009) 262302 [arXiv:0901.4588] [INSPIRE].

[14] M. Gyulassy, I. Vitev, X.-N. Wang and P. Huovinen, Transverse expansion and high $p_{T}$ azimuthal asymmetry at RHIC, Phys. Lett. B 526 (2002) 301 [nucl-th/0109063] [INSPIRE].

[15] M. Gyulassy, I. Vitev and X.-N. Wang, High $p_{T}$ azimuthal asymmetry in noncentral $A+A$ at RHIC, Phys. Rev. Lett. 86 (2001) 2537 [nucl-th/0012092] [INSPIRE].

[17] ALICE collaboration, Centrality dependence of charged particle production at large transverse momentum in Pb-Pb collisions at $\sqrt{s_{\mathrm{NN}}}=2.76 \mathrm{TeV}$, Phys. Lett. B 720 (2013) 52 [arXiv:1208.2711] [INSPIRE].

[18] ALICE collaboration, Anisotropic flow of charged hadrons, pions and (anti-)protons measured at high transverse momentum in $\mathrm{Pb}-\mathrm{Pb}$ collisions at $\sqrt{s_{\mathrm{NN}}}=2.76 \mathrm{TeV}$, Phys. Lett. B 719 (2013) 18 [arXiv: 1205.5761] [INSPIRE].

[19] CMS collaboration, Study of high- $p_{T}$ charged particle suppression in $\mathrm{PbPb}$ compared to $\mathrm{pp}$ collisions at $\sqrt{s_{\mathrm{NN}}}=2.76 \mathrm{TeV}$, Eur. Phys. J. C 72 (2012) 1945 [arXiv:1202.2554] [INSPIRE].

[20] CMS collaboration, Measurement of the azimuthal anisotropy of neutral pions in $\mathrm{Pb}-\mathrm{Pb}$ collisions at $\sqrt{s_{\mathrm{NN}}}=2.76 \mathrm{TeV}$, Phys. Rev. Lett. 110 (2013) 042301 [arXiv:1208.2470] [INSPIRE].

[21] ATLAS collaboration, Measurement of the azimuthal anisotropy for charged particle production in $\sqrt{s_{\mathrm{NN}}}=2.76 \mathrm{TeV}$ lead-lead collisions with the ATLAS detector, Phys. Rev. C 86 (2012) 014907 [arXiv: 1203.3087] [INSPIRE].

[22] ATLAS collaboration, Measurement of the pseudorapidity and transverse momentum dependence of the elliptic flow of charged particles in lead-lead collisions at $\sqrt{s_{\mathrm{NN}}}=2.76 \mathrm{TeV}$ with the ATLAS detector, Phys. Lett. B 707 (2012) 330 [arXiv:1108.6018] [INSPIRE].

[23] J. Liao and E. Shuryak, Angular dependence of jet quenching indicates its strong enhancement near the QCD phase transition, Phys. Rev. Lett. 102 (2009) 202302 [arXiv:0810.4116] [INSPIRE].

[24] X. Zhang and J. Liao, Hard probe of geometry and fluctuations in heavy ion collisions at $\sqrt{s_{\mathrm{NN}}}=0.2,2.76$ and 5.5 TeV, Phys. Rev. C 89 (2014) 014907 [arXiv:1208.6361] [InSPIRE].

[25] X. Zhang and J. Liao, Event-by-event azimuthal anisotropy of jet quenching in relativistic heavy ion collisions, Phys. Rev. C 87 (2013) 044910 [arXiv: 1210.1245] [INSPIRE]. 